\title{
SECANT FUNCTIONS, THE REISS RELATION AND ITS CONVERSE
}

BY

MARK L. GREEN ${ }^{1}$

\begin{abstract}
Generalizing a classical Euclidean theorem for the circle, certain meromorphic functions on $\mathbf{P}_{1}$ relating to the geometry of algebraic plane curves are shown to be constant. Differentiated twice, this gives a new proof of the Reiss relation and its converse. The relation of these functions to Abel's Theorem is discussed, and a generalization of secant functions to space curves is given, for which the Chow form arises in a natural way.
\end{abstract}

1. Introduction-secants of the circle. The purpose of this note is to introduce certain new functions of a variable linear space called secant functions which appear to be useful in studying the geometry of projective varieties. One application is a particularly nice proof of the converse of the Reiss relation for plane curves in $\$ 4$ (see [2] for another proof). We also hope that they will be useful in studying space curves when combined with Cayley's equations for the Chow variety of space curves (see [1]) as indicated in $\$ 5$, and that they may shed light on questions related to Abel's Theorem, as indicated in $§ 3$.

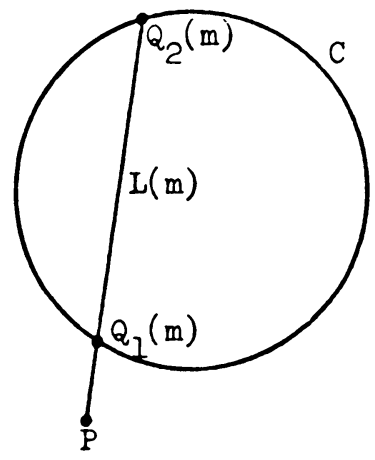

A good illustration of secant functions is the following new proof of an extremely old theorem. Let $C$ be a circle and $P$ an exterior point. Let $L(m)$ be the line through $P$ having slope $m$ and meeting $C$ in points $Q_{1}(m)$ and $Q_{2}(m)$. Then

$$
d\left(P, Q_{1}(m)\right) \cdot d\left(P, Q_{2}(m)\right)=\text { constant }
$$

Received by the editors March 26, 1982.

1980 Mathematics Subject Classification. Primary 14N05, 53Axx.

${ }^{1}$ Research partially supported by N.S.F. Grant MCS 82-00924.

(C)1983 American Mathematical Society $0002-9947 / 83 \$ 1.00+\$ .25$ per page 
where $d$ denotes distance. To prove this, take $P=(0,0)$ and $Q_{i}(m)=\left(x_{i}(m), y_{i}(m)\right)$, $i=1,2$. Let

$$
u(m)=\prod_{i=1}^{2}\left(x_{i}(m)^{2}+y_{i}(m)^{2}\right) .
$$

We want to show that $u$ is constant. Work over the complex numbers, so that $m \in \mathbf{C P}_{1}=$ the pencil of lines through $P$; since $y_{i}(m)=m x_{i}(m)$, we have

$$
u(m)=\left(m^{2}+1\right)^{2} \prod_{i=1}^{m} x_{i}(m)^{2} .
$$

Now $u(m)$ is regular at $m$ except when $\left(x_{i}(m), y_{i}(m)\right)$ is one of the points at infinity of $C$. For circles, these occur when $m= \pm i$ and give $x_{i}(m)$ a simple pole. These are cancelled by the zeros of $m^{2}+1$, so that $u$ is a regular function at all points of $\mathbf{P}_{1}$ and, hence,

$$
u(m)=\text { constant. }
$$

Somewhat more generally, if $P=(a, b)$ and

$$
u(P, m)=\prod_{i=1}^{2}\left(\left(x_{i}(m)-a\right)^{2}+\left(y_{i}(m)-b\right)^{2}\right),
$$

then $u$ is constant in $m$ and vanishes (to order 2) if and only if $P \in C$. Thus,

$$
u(P, m)=\text { constant } \cdot g(p)^{2}
$$

where $C=\{g(x, y)=0\}$. Note that the secant function $u$ gives a way of explicitly reconstructing $C$ from information about how $C$ meets a variable line.

2. Secant functions for plane curves. Let $C=\left\{G\left(z_{0}, z_{1}, z_{2}\right)=0\right\}$, deg $G=d$, be a plane curve. We generalize from using a pencil of lines, as in the last section, to a pencil of curves $P_{1}+m P_{2}$ of degree $k$, and from the line at infinity to a curve $F$ of degree $l$. Let

$$
\left(P_{1}+m P_{2}\right) \cap C=Q_{1}(m)+\cdots+Q_{d k}(m)
$$

and

$$
F \cap C=E_{1}+\cdots+E_{d l},
$$

taking algebraic intersection in both cases. Define numbers $m_{j}$ by

$$
P_{1}\left(E_{j}\right)+m_{j} P_{2}\left(E_{j}\right)=0, \quad j=1, \ldots, d l .
$$

Define the secant function of $C$ relative to the curve $F$ and the pencil $P_{1}+m P_{2}$,

$$
u(m)=\left(\prod_{j=1}^{d l}\left(m_{j}-m\right)^{k}\right)\left(\prod_{i=1}^{d k} \frac{P_{2}\left(Q_{i}(m)\right)^{l}}{F\left(Q_{i}(m)\right)^{k}}\right) .
$$

Note that since

$$
P_{1}\left(Q_{i}(m)\right)+m P_{2}\left(Q_{i}(m)\right)=0
$$


we have that

$$
\begin{aligned}
u(m)^{d} & =\left(\prod_{j=1}^{d l}\left(m_{j}-m\right)^{d k}\right)\left(\prod_{i=1}^{d k} \frac{P_{2}\left(Q_{i}(m)\right)^{d l}}{F\left(Q_{i}(m)\right)^{d k}}\right) \\
& =\frac{\prod_{j=1}^{d l} \prod_{i=1}^{d k}\left(m_{j}-m\right) P_{2}\left(Q_{i}(m)\right)}{\prod_{i=1}^{d k} F\left(Q_{i}(m)\right)^{d k}} \\
& =\frac{\prod_{j=1}^{d l} \prod_{i=1}^{d k}\left(P_{1}\left(Q_{i}(m)\right)+m_{j} P_{2}\left(Q_{i}(m)\right)\right)}{\prod_{i=1}^{d k} F\left(Q_{i}(m)\right)^{d k}}
\end{aligned}
$$

So

$$
u(m)^{d}=\prod_{i=1}^{d k}\left(\prod_{j=1}^{d l} \frac{\left(P_{1}\left(Q_{i}(m)\right)+m_{j} P_{2}\left(Q_{i}(m)\right)\right)}{F\left(Q_{i}(m)\right)^{d k}}\right)
$$

which is analogous to the secant function considered in $\S 1$.

Returning to the formula (2.4) for $u(m)$, we see that the first term vanishes to order $k$ whenever $m=m_{j}$, and this cancels the zero of order $k$ of $F\left(Q_{i}(m)\right)^{k}$ when $m=m_{j}$ in the denominator. The first term has a pole of order $d k l$ at $m=\infty$, which is cancelled by the fact that $P_{2}\left(Q_{i}(\infty)\right)^{l}=0$ to order $l$ for each $i=1, \ldots, d k$. Thus $u(m)$ is a regular function everywhere on $\mathbf{P}_{1}$ and thus,

$$
u(m)=\text { constant. }
$$

This also follows from a reciprocity formula of Weil (cf. [3, p. 242]).

If we take $F=z_{0}, P_{1}=z_{2}-b z_{0}$ and $P_{2}=a z_{0}-z_{1}$, then by (2.4),

$$
u(m)=\left(\prod_{j=1}^{d}\left(m_{j}-m\right)\right)\left(\prod_{i=1}^{d}\left(x_{i}(m)-a\right)\right)
$$

and by (2.6),

$$
u(m)^{d}=\prod_{i=1}^{d} \prod_{j=1}^{d}\left(m_{j}\left(x_{i}(m)-a\right)-\left(y_{i}(m)-b\right)\right) .
$$

If we write $u(m)$ as $u(m, a, b)$, then

$$
u(m, a, b)=(\text { constant }) g(a, b)
$$

by the same argument as for (1.6), where $g(x, y)$ is the affinization of $G\left(z_{0}, z_{1}, z_{2}\right)$.

3. Abel's Theorem. The relation between secant functions and Abel's Theorem is simplest in the case of a triangle, i.e. three lines $L_{1}, L_{2}, L_{3}$ in general position in $\mathbf{P}_{2}$. A plane curve $C$ of degree $d$ meets each side of the triangle in $d$ points. Given $3 d$ points on a triangle, $d$ on each side, the problem addressed by Abel's Theorem is under what conditions are they a complete intersection, i.e. the intersection of the triangle with a plane curve of degree $d$ ? 


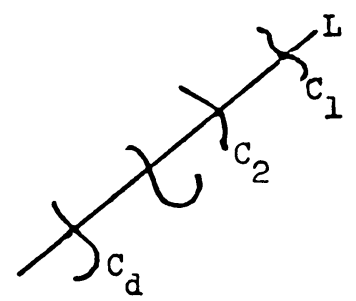

Abel's TheOREM FOR A TRIANgLe (SEE [2]). There is exactly one relation necessary and sufficient for $3 d$ points on a triangle, $d$ on each side, to be a complete intersection.

Proof. Take $L_{3}$ to be the line of infinity, and $L_{1}$ and $L_{2}$ the $x$ and $y$ axes. From knowing the $d$ points of $C \cdot L_{3}$, we know $m_{1}, \ldots, m_{d}$ and thus can commute $u(0)$ and $u(\infty)$ for the secant function $u$ of $C$ relative to the curve $L_{3}$ and the pencil $L_{1}+m L_{2}$. The relation

$$
u(0)=u(\infty)
$$

is clearly necessary if we are to have a complete intersection.

Conversely, by (2.10), we can compute what should be the restrictions of $G$ to $L_{1}, L_{2}$ and $L_{3}$ up to multiplication by unknown constants $c_{1}, c_{2}, c_{3}$, respectively, by using the secant functions for pencils of lines whose base point lies on the triangle. The necessary condition (3.1) says that we can adjust the constants $c_{1}, c_{2}, c_{3}$ so that the functions agree at the three vertices. Thus we obtain, in a fairly explicit way, a section

$$
\bar{G} \in H^{0}\left(L_{1} \cup L_{2} \cup L_{3}, O(d)\right)
$$

whose divisor is the $3 d$ given points. From the exact sequence

$$
0 \rightarrow \vartheta_{\mathbf{P}_{2}}(d-3) \rightarrow \vartheta_{\mathbf{P}_{2}}(d) \rightarrow \vartheta_{L_{1} \cup L_{2} \cup L_{3}}(d) \rightarrow 0
$$

and the fact that

$$
H^{1}\left(\mathbf{P}_{2}, \vartheta_{\mathbf{P}_{2}}(d-3)\right)=0,
$$

we conclude that $\bar{G}$ extends to a homogeneous polynomial $G \in H^{0}\left(\mathbf{P}_{2}, \vartheta_{\mathbf{P}_{2}}(d)\right)$ and thus the condition (3.1) is sufficient.

More generally, let $C=\{g(x, y)=0\}$ be a plane curve of degree $d$ and let $Q_{1}, \ldots, Q_{d k}$ be $d k$ points on $C$. When does there exist a curve $D=\{f(x, y)=0\}$ meeting $C$ in $Q_{1}, \ldots, Q_{d k}$ ? Abel's Theorem states that there are precisely genus $(C)$ conditions on $Q_{1}, \ldots, Q_{d k}$ necessary and sufficient for this to happen.

Let $L=\{y=m x+b\}$ be a variable line. Let

$$
L \cdot C=P_{1}+\cdots+P_{d} .
$$

Let $L_{\infty}$ be the line at infinity and

$$
L_{\infty} \cdot C=S_{1}+\cdots+S_{d} .
$$

Set

$$
u(L)=\frac{\prod_{j=1}^{d k} L\left(Q_{j}\right)}{\prod_{l=1}^{d} L\left(S_{l}\right)^{k}}
$$


where we identify $L$ with $y-m x-b$. Note that $u$ vanishes when $Q_{j} \in L$ for some $h$ and has a pole of order $k$ when $S_{l} \in L$ for some $l$. The same is true for the function $\prod_{i=1}^{d} f\left(P_{i}\right)$ and thus

$$
u(L)=\text { constant } \cdot \prod_{l=1}^{d} f\left(P_{i}\right) .
$$

The secant function $u$ may be calculated from the data of $C$ and $Q_{1}, \ldots, Q_{d k}$. The problem of recovering $f$ from $u$ has a certain formal analogy to the Radon transform, for if we take $\operatorname{logarithms}$ then $\frac{1}{d} \log u$ is the "average" of $\log f$ over lines. The function $u$ is defined whether $f$ exists or not, and not every function on lines is of the form $\prod_{P \in L \cdot C} f(P)$. It would be interesting to see how the conditions on $Q_{1}, \ldots, Q_{d k}$ that arise in this way relate to those of Abel's Theorem.

4. The Reiss relation and its converse. For a real plane curve given implicitly as $f(x, y)=0$, the curvature $k$ is given by

$$
k=\frac{-f_{x x} f_{y}^{2}+2 f_{x y} f_{x} f_{y}-f_{y y} f_{x}^{2}}{\left(f_{x}^{2}+f_{y}^{2}\right)^{3 / 2}}
$$

where the curve is suitably oriented. In the case of a complex plane curve in $\mathbf{C P}_{2}$, the right-hand side of (4.1) continues to make sense (except for a choice of sign coming from the square root) and will be called the formal curvature of the curve. If the line $a x+b y=c, a^{2}+b^{2}=1$, meets the curve $f(x, y)=0$ and their tangents make an angle $\theta$, then

$$
\sin \theta=\frac{a f_{y}-b f_{x}}{\left(f_{x}^{2}+f_{y}^{2}\right)^{1 / 2}}
$$

for a suitable choice of orientation. The equation

$$
\frac{k}{\sin ^{3} \theta}=\frac{-f_{x x} f_{y}^{2}+2 f_{x y} f_{x} f_{y}-f_{y y} f_{x}^{2}}{\left(a f_{y}-b f_{x}\right)^{3}}
$$

is true in the real case and constitutes a formal definition of $k / \sin ^{3} \theta$ in the complex case.

The Reiss Relation (SEE [3]). Let $C=\{f(x, y)=0\}$ be an algebraic plane curve of degree $d$, and $L$ a line meeting $C$ transversally in the $d$ points $P_{1}, \ldots, P_{d}$. Then

$$
\sum_{i=1}^{d} \frac{k}{\sin ^{3} \theta}\left(P_{i}\right)=0 .
$$

There is a startling converse to the Reiss relation (see [3]).

Converse to the Reiss relation. Given a line $L$ in $\mathbf{P}_{2}$, and $d$ little complex analytic arcs $C_{1}, \ldots, C_{d}$ meeting $L$, if the Reiss relation (4.4) holds for all lines in a neighborhood of $L$ in $\mathbf{P}_{2}^{*}$, then there exists an algebraic plane curve $C$ of degree $d$ that agrees with the arcs $C_{1}, \ldots, C_{d}$ on a neighborhood of $L$ in $\mathbf{P}_{2}$.

These two results have a nice proof using secant functions. 


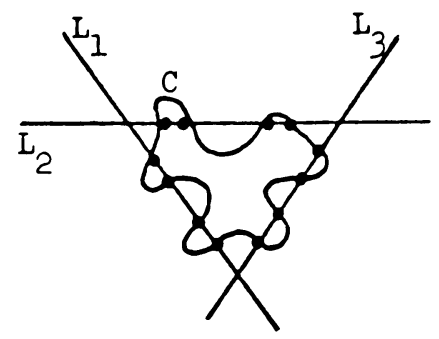

Proof of the Reiss relation. Take $L$ to be the line $y=0$. Let $L(m)$ be the line through $(a, b)$ of slope $m$ and

$$
\begin{aligned}
& L \cdot C=P_{1}(m, a, b)+\cdots+P_{d}(m, a, b), \\
& P_{i}(m, a, b)=\left(x_{i}(m, a, b), y_{i}(m, a, b)\right) .
\end{aligned}
$$

Let $m_{1}, \ldots, m_{d}$ be the slopes of the points at $\infty$ of $C$. Then the secant function of $C$, relative to the line at $\infty$ and the pencil $y+m x$,

$$
u(m)=\prod_{i=1}^{d}\left(m-m_{i}\right)\left(x_{i}(m, a, b)-a\right),
$$

is constant in $m$. So

$$
0=\frac{\partial \log u}{\partial m}=\sum_{i=1}^{d} \frac{1}{m-m_{i}}+\sum_{i=1}^{d} \frac{\partial x_{i} / \partial m}{x_{i}-a} .
$$

At $m=0,(4.6)$ becomes

$$
0=\frac{\partial \log u}{\partial m}=\sum_{i=1}^{d} \frac{\partial x_{i} / \partial m}{x_{i}-a}-\sum_{i=1}^{d} \frac{1}{m_{i}} .
$$

Since

$$
y_{i}(m, a, b)-b=m\left(x_{i}(m, a, b)-a\right)
$$

we have that

$$
\frac{\partial y_{i}}{\partial m}=x_{i}-a+m \frac{\partial x_{i}}{\partial m}
$$

so at $m=0$,

$$
\frac{\partial y_{i}}{\partial m}=x_{i}-a .
$$

Thus, combining (4.7) and (4.10), we have

$$
\begin{aligned}
0 & =\frac{\partial \log u}{\partial m}=\sum_{i=1}^{d} \frac{\partial x_{i} / \partial m}{\partial y_{i} / \partial m}-\sum_{i=1}^{d} \frac{1}{m_{i}} \\
& =\sum_{i=1}^{d}\left(\frac{d x}{d y}\right)\left(P_{i}(m, a, b)\right)-\sum_{i=1}^{d} \frac{1}{m_{i}} .
\end{aligned}
$$


Differentiating with respect to $m$ and noting that when $m=0, \partial y_{i}(m, a, b) / \partial b=1$, we get

$$
0=\frac{\partial^{2} \log u}{\partial m \partial l}=\sum_{i=1}^{d} \frac{d^{2} x}{d y^{2}}\left(P_{i}(m, a, b)\right) .
$$

However, by calculus,

$$
\frac{d^{2} x}{d y^{2}}=\frac{-f_{x x} f_{y}^{2}+2 f_{x y} f_{x} f_{y}-f_{y y} f_{x}^{2}}{f_{x}^{3}}=\frac{k}{\sin ^{3} \theta}
$$

so (4.12) is the Reiss relation.

Proof of the Converse of the Reiss relation. Let $L$ be the line at infinity $L_{\infty}$ and assume the Reiss relation is true on a neighborhood $U^{*}$ of $L$ in $\mathbf{P}_{2}^{*}$. This neighborhood includes all lines with $m=0$ and sufficiently large $y$-intercept. Let $u(m, a, b)$ be defined by (4.5) for all values of $(m, a, b)$ corresponding to lines in $U^{*}$. If $L^{\#}$ is a line with $m=0$, then the preceding calculation shows that

$$
\text { Reiss relation for } L^{\sharp} \leftrightarrow \sum_{i=1}^{d} \frac{d^{2} x}{d y^{2}}\left(P_{i}(m, a, b)\right)=0 \quad \text { at } L^{\#} \text {. }
$$

Thus,

$$
\sum_{i=1}^{d} \frac{d x}{d y}\left(P_{i}(m, a, b)\right)=\text { constant }
$$

for lines with slope 0 sufficiently near the line at infinity. Taking a limit as the line goes to infinity, we see that for $L_{\infty}$ we have

$$
\sum_{i=1}^{d} \frac{d x}{d y}\left(P_{i}(m, a, b)\right)=\sum_{i=1}^{d} \frac{1}{m_{i}}
$$

and, thus, this is true for all lines with $m=0$ in $U^{*}$. By (4.11) this is equivalent to

$$
\partial \log u / \partial m=0 \text {. }
$$

This being a coordinate-free equation, it is also true for lines of slopes $\neq 0$ by a change of coordinates. Thus,

$$
u(m, a, b)=g(a, b), \quad(a, b) \in U,
$$

for some meromorphic function $g$ where $U=\left\{(a, b) \in \mathbf{P}_{2} \mid\right.$ some line through $(a, b)$ belongs to $\left.U^{*}\right\}$. Since $g$ is holomorphic on $U-L_{\infty}$ by (4.5), and has a pole of order $d$ on $L_{\infty}$, we conclude by Hartog's theorem and Chow's theorem that $g$ extends to a polynomial of degree $d$. By (4.5), the zeros of $g$ inside $U$ are just the little arcs we started with, and this completes the proof.

5. Secant functions for space curves. Let $C$ be a curve in $\mathbf{P}_{3}$ of degree $d$. Let $L=H_{1} \cap H_{2}$ be a line in $\mathbf{P}_{3}$, where

$$
H_{1}=x-\alpha z-\beta, \quad H_{2}=y-\gamma z-\delta .
$$

Let

$$
\left(H_{1}+m H_{2}\right) \cdot C=P_{1}(m)+\cdots+P_{d}(m)
$$


and let

$$
H_{\infty} \cdot C=Q_{1}+\cdots+Q_{d},
$$

where $H_{\infty}$ is the hyperplane at infinity. Define $m_{1}, \ldots, m_{d}$ by

$$
H_{1}\left(Q_{i}\right)+m_{i} H_{2}\left(Q_{i}\right)=0 .
$$

We now introduce the secant function

$$
u(m)=\prod_{i=1}^{d}\left(H_{1}\left(P_{i}(m)\right)+m_{i} H_{2}\left(P_{i}(m)\right)\right)
$$

which, by (5.2), has the alternate expression

$$
u(m)=\prod_{i=1}^{d}\left(m_{i}-m\right) H_{2}\left(P_{i}(m)\right) .
$$

As occurs for the secant functions already discussed, the poles of $H_{2}\left(P_{i}(m)\right)$ exactly cancel the zeros of $m_{i}-m$, so that $u$ is constant in $m$. If we now write $u$ as $u(m, L)$, then

$$
u(m, L)=0 \leftrightarrow L \cap C \neq \varnothing .
$$

If $C_{0}=\{z=0\} \cap H_{\infty}$ then

$$
u(m, L)=\infty \leftrightarrow L \cap C_{0} \neq \varnothing
$$

and this occurs to order $d$. Thus,

$$
u(m, L)=\text { constant } \cdot\left(F_{C} / F_{C_{0}}^{d}\right)
$$

where $F_{C}$ and $F_{C_{0}}$ are the Chow forms of $C$ and $C_{0}$, respectively.

Formula (5.9) may be used in the following way. Let $H$ be a hyperplane in $\mathbf{P}_{3}$ and let $W_{H}=\left\{\right.$ lines $\left.L \subset \mathbf{P}_{3} \mid L \subset H\right\} \subset G(2,4)$. Then

$$
C \cdot H \text { determines }\left.F_{C}\right|_{W_{H}} \text { up to a constant; }
$$

as for any $L \subset H$, we can determine $u(m, L)$ for one value of $m$ by evaluating it for $H$.

Let $H_{1}, \ldots, H_{k}$ be hyperplanes in $\mathbf{P}_{3}$, and let $Q_{1}^{i}, \ldots, Q_{d}^{i} \in H_{i}$ for $i=1, \ldots, k$. We obtain a prescription for whether there exists a curve $C$ of degree $d$ in $\mathbf{P}_{3}$ so that

$$
C \cdot H_{i}=Q_{1}^{i}+\cdots+Q_{d}^{i}, \quad i=1, \ldots, k,
$$

as follows:

(1) Construct $\left.F_{C}\right|_{W_{H_{i}}}$ up to a constant for each $i$, which is possible by (5.10).

(2) Check whether the constants can be adjusted so that we obtain a candidate for $\left.F_{C}\right|_{W_{H_{1}}}+\cdots+w_{H_{k}} \cdot$

(3) Now adjust by polynomials of degree $d$ in the Plücker coordinates vanishing on $W_{H_{1}}+\cdots+W_{H_{k}}$ so that the conditions for $F_{C}$ to be a Chow form are satisfied (using the equations of [1]). 
RemarKs. Step (2) is analogous to what was done in $\$ 3$ for Abel's Theorem for a Triangle. The conditions of step (3) are new, an additional difficulty that comes from working in codimension two. These conditions seem impractical to verify, but ought, perhaps, to point the way to something better.

\section{BIBLIOGRAPHY}

I. M. Green and I. Morrison, The equations defining Chow varieties, Duke Math. J. (to appear).

2. P. Griffiths, Variations on a theorem of Abel, Invent Math. 35 (1976), 321-390.

3. P. Griffiths and J. Harris, Principles of algebraic geometry, Wiley, New York, 1978.

Department of Mathematics, University of California, Los Angeles, California 90024 